WS9-C07

\title{
Assimilation of Time-lapse CSEM Data for Fluid
} Flow Monitoring

M. Lien* (Octio AS), T. Mannseth (Uni CIPR) \& R. Agersborg (Octio AS)

\section{SUMMARY}

The feasibility of marine CSEM for reservoir monitoring is supported by several model studies suggesting that there may be a detectable time-lapse signal in CSEM data. However, the detectability of these signals for field cases will depend on careful acquisition design and reliable inversion methodology. To mitigate the inherent non-uniqueness associated with these large scale geophysical problems, the integration of additional data types is required. Constraining the solution space by including different data types in the model calibration process can help reducing the model uncertainty and lower production related risk. In this talk, the use of statistical ensemble based methods (EnKF) for the integration of CSEM data and other geophysical data types (i.e. gravimetric data) will be discussed. By the utilization of a statistical inversion method, an assessment of the uncertainty in the estimated flow patterns is obtained as part of the solution. 


\section{Amsterdam ' 14}

\section{Introduction}

Marine CSEM data has proven its value for exploration of hydrocarbon reservoirs. The use of marine CSEM data for monitoring is still a subject of debate. Though shown to be a reliable hydrocarbon indicator, there are several challenges associated with the interpretation of CSEM data that may tamper its use for fluid flow monitoring. Of special importance will be to secure necessary time-lapse repeatability in the data, reducing errors from uncertainties in the source and receiver positioning and in the modelling of the surrounding environment.

For the description of complex systems like offshore reservoirs, integration of all relevant information will be deciding to obtain robust and reliable results within acceptable uncertainty bounds. Integration of different geophysical data for reservoir characterization and monitoring has mainly been focused on combining seismic and electromagnetic data. Gravity data is currently emerging as an additional and independent source of information after proving its value through numerous gas monitoring projects in the North Sea (Glegola, Ditmar, Hanea, Vossepoel, Arts, \& Klees, 2012; Fageraas, Lien, \& Agersborg, 2013). By providing a direct measure of the density profile within the reservoir, gravity data can represent a valuable source of information for the estimation of fluid flow.

There is an ongoing discussion as to what is the optimal approach for integrating different, possibly disparate data types. Many algorithms have been developed, ranging from loosely coupled, sequential integration of different data types, to more strongly coupled inversion where all data types are inverted for simultaneously. A strong coupling of the different data types corresponds to putting strict constraints on the problem, possibly introducing bias in the final estimates. A too loose coupling on the other hand, can render the problem ill-posed with associated non-uniqueness and instability issues.

For risk assessment, a measure of the uncertainty in the final flow estimates is important. A main advantage with the use of statistical inversion methods is that a measure of the uncertainty in the final models is obtained as part of the solution. If the mapping from reservoir properties to the measured data is nonlinear, the posterior pdf can only be assessed through sampling. Markov chain Monte Carlo methods sample correctly from the posterior pdf. For large scale geophysical problems, however, they quickly become too numerically demanding. For complex models with multiple model parameters, the use of "approximate" sampling methods like Randomized Maximum Likelihood or the Ensemble Kalman Filter (EnKF) can be used.

In this talk, the use of statistical ensemble based methods (EnKF) for the integration of CSEM data and other geophysical data types (i.e. gravimetric data) will be discussed. For increased time-lapse repeatability we will discuss the use of (semi)-permanent seafloor stations, see Figure 1 showing a typical survey layout for a time-lapse gravimetric survey.

\section{Assimilation of time-lapse CSEM data for fluid flow monitoring}

Several model studies suggest that there may be a detectable time-lapse signal in CSEM data for monitoring oil and gas reservoirs. However, the detectability and amount of information that can be retrieved from these signals depend on careful acquisition design and reliable inversion methodology.

In (Lien, 2013) an approach for joint inversion of seismic AVO and CSEM data for fluid flow monitoring is presented based on a re-parameterization of the model parameters inspired by the levelset method. With this approach the data can be coupled both through the estimation of common parameter structure (i.e. the fluid front) and using a joint relation to fluid saturation by means of rock physics modeling. In this talk, we will show how this approach can be extended to also include marine gravity data. Gravity data has shown to provide important information related to reservoir performance such as the volume of available reserves, fluid flow, and water influx, motivating its use as an integrated part of reservoir monitoring programs. 
The Ensemble Kalman Filter is an ensemble based approximate sampling method based on the sequential Bayesian formulation, where the data is assimilated as it becomes available. Encouraging results from many synthetic studies have led to increased popularity of the method and stimulated the use of EnKF for real-field cases.

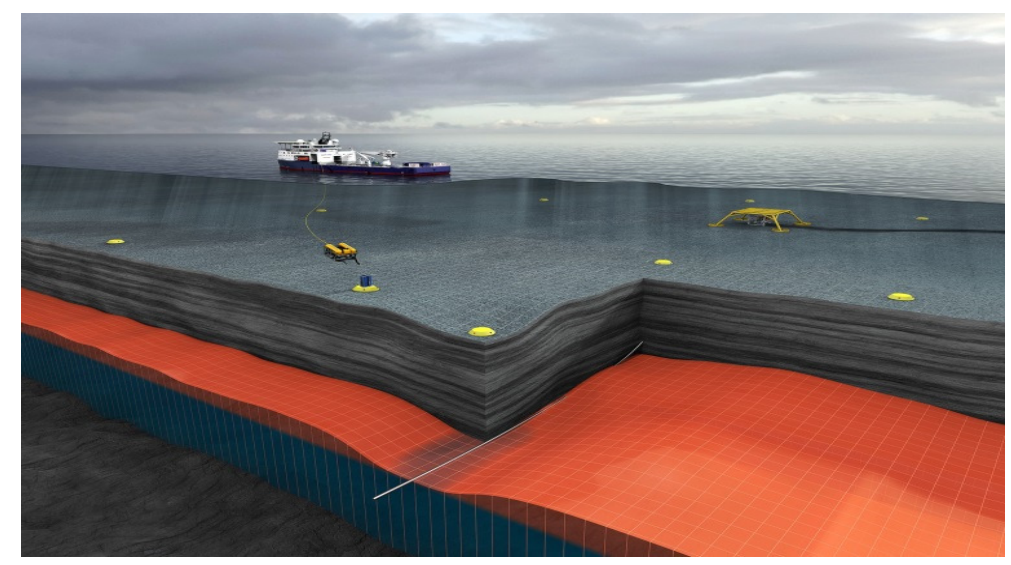

Figure 1 Semi-permanent seafloor stations, serving as reference benchmarks for time-lapse geophysical surveys.

A main assumption underlying EnKF is that all the prior probability distributions are Gaussian. For a representation of realistic flow scenarios following a bi-modal distribution, this assumption will be violated. To enable the identification of possibly multi-model parameter fields different modifications of the traditional EnKF method have been developed. Here the different approaches will be briefly described, including also a discussion on how the re-parameterisation utilized in (Lien, 2013) can be applied for fluid flow monitoring within the EnKF framework following the ideas presented in (Lien \& Mannseth, 2013).

\section{Conclusions}

Modelling studies indicate that there may be a detectable time-lapse signal in CSEM data for reservoir monitoring. However, the integration of additional data types is required to mitigate the inherent non-uniqueness associated with these large scale geophysical problems. By including different data types in the model calibration process, the solution space is constrained which can reduce the model uncertainty and lower production related risk. In the current talk, methods for combining CSEM data with seismic and gravity data for reservoir monitoring within a statistical framework is discussed. By the utilization of a statistical inversion method, an assessment of the uncertainty in the estimated flow patterns is obtained as part of the solution.

\section{References}

Fageraas, B., Lien, M. and Agersborg, R. [2013] Using gravity to enhance recovery. GeoExpro, 62-65.

Glegola, M., Ditmar, P., Hanea, R., Vossepoel, F., Arts, R. and Klees, R. [2012] Gravimetric monitoring of water influx into a gas reservoir: A numerical study based on the ensemble kalman filter. SPE Journal, SPE-149578-PA.

Lien, M. [2013] Simultanous joint inversion of amplitude-vesus-offset and controlled-source electromagnetic data by implicit representation of common parameter structure. Geophysics, p. 1-13.

Lien, M. and Mannseth, T. [2013]. Facies estimation through data assimilation and structure parameterization. Submitted. 